\title{
Faktorisierung eines Differentialoperators unendlicher Ordnung in verallgemeinerten Ableitungen ${ }^{2}$ ).
}

\section{A. Tumófeev .}

Es wird das Faktorisierungsproblem für den Differentialoperator gelöst, der 1965 von A. F. Leontjev eingeführt wurde und der dẹn Differentialoperator von Gelfond-Leontjev verallgemeinert.

Решается задача факторизации для дифференциального оператора, введенного А. Ф. Леонтьевым в 1965 году. и .обобшающего оператор Гельфонда-Јеонтьева.

The problem of factorization is solved for the differential operator, originally presented by A. F. Leontjev in 1965 and generalizing the operator by Gelfond-Leontjev.

0. Einleitung. Es sei die gewöhnliche Differentialgleichung endlicher Ordnung

$$
\sum_{k=0}^{N} c_{k} y^{(k)}(z)=0
$$

mit dem charakteristischen Polynom $P, P(\lambda)=c_{0}+c_{1} \lambda+\cdots+c_{N} \lambda^{N}$, gegeben. Setzen wir voraus, da $B . P=P_{1} P_{2}$ ist, wobei die Polynome $P_{1}$ und $P_{2}$ keine gemeinsamen Nullstellen haben dürfen, dann kann man jecle Lösung $y$ der Gleichung (1) in der Form $y=y_{1}+y_{2}$ darstellen, wobei $y_{1}$ und $y_{2}$ die Lösung von (1) mit dem charakteristischen Polynom $P_{1}$ und $P_{2}$ ist. Ein solches Falitorisierungsproblem. wurde.von Hadamard für die partielle Differentialgleichung endlicher Ordnung ,

$$
\sum_{|\alpha|=0}^{N} c_{\alpha} D^{\alpha} y=0 \quad \text { mit } \quad \cdot D^{\alpha} y=\partial^{|\alpha|} y / \partial x_{1}{ }^{\alpha_{\iota}} \ldots \partial x_{n}{ }^{\alpha_{n}}
$$

formuliert und in [6] von Matsúra gelöst. Die Beiträge [3, 8-10] beschäftigten sich mit dem Faltorisierungsproblem für Differentialoperatoren unendlicher Ordnung in gewöhnlichen Ableitungen bzw. in Ableitungen von Gelfond-Leontjev. Es wurde gezeigt, daß in cliesen Fällen im Unterschied zu Differentialoperatoren endlicher Ordnung nicht notwendig der Satz ïber die additive Zerlegung der Lösung gilt. In diesem Beitrag wird das Faktorisierungsproblem für den von LeontJev [2] eingeführten Differentialoperator gelöst. Dieser Operator verallgemeinert den Differentialoperator von Gelfond-I.eontjev.

1. Formulierung des Hauptsatzes. Wir bezeichnen mit $\left[\varrho_{1}, \infty\right)$ den Raum der ganzen Funktionen von der Ordnung $\varrho_{1}$, die einen beliebigen endlichen Typ besitzen. Es ist bekannt, da $B\left[\varrho_{1}, \infty\right)=\underset{0<t<\infty}{\lim }$ ind $B_{\mathrm{r}}$ mit

$B_{\mathrm{r}}=\left\{F \in H(\mathbb{C}):\|F\|_{\mathrm{r}}=\sup _{0<r<\infty} M(F, r) \exp \left[-\tau r^{\boldsymbol{Q}_{1}}\right]<\infty\right\}, \quad M(F, r)=\max _{|z|=r}|F(z)|$ ist. Es sei $f(z)=\sum a_{n} z^{n}$ eine ganze Funktion mit positiven Koeffizienten, die der Be-

1) Der Autor dankt Prof. Dr. W. Tutschke und Dr. M. Reissig für die Hilfe bei der Fertigstellung des Beitrages. 
dingung $n^{1 / e} \sqrt[n]{a_{n}} \rightarrow(\sigma e \varrho)^{1 / e}$ mit $0<\varrho<\varrho_{1}$ für $n \rightarrow \infty$ genügt. Der Differentialoperator von Gelfond-Leontjev $D$, wird auf folgende Weise definiert:

$$
D_{f} F(z)=\sum_{k=0}^{\infty} b_{k+1} \frac{a_{k}}{a_{k+1}} z^{k} \quad \text { für } \quad F(z)=\sum_{k=0}^{\infty} b_{k} z^{k}
$$

Für $f(z)=\mathrm{e}^{z}$ erhalten wir den gewöhnlichen Differentialoperator: $D F=F^{\prime}$.

Es sei ein System ganzer Funktionen $P=\left\{P_{n}\right\}_{n=0}^{\infty}$ mit folgenden Eigenschaften gegeben:

1. ' $\left|P_{n}(z)\right| \leqq C a_{n}(1+|\dot{z}|)^{n} \exp \left(B|z|^{\alpha}\right)$ für ein gewisses $\alpha \leqq \varrho_{1}$

und positive Konstanten $C$ und $B$.

2. Jede Funktion $F \in\left[\varrho_{1}, \infty\right)$ ist in der ganzen Ebene $\mathbb{C}$ in der Form $F(z)$ $=\sum d_{k} P_{k}(z)$ darstellbar, wobei

$$
\left|a_{n} d_{n}\right|<c_{0} R^{-n}, M(F, \gamma R) \quad \text { für jedes } R>R_{0} \text { und } n \in \mathbb{N}_{0}
$$

gilt. Die positiven Konstanten $c_{0}$ und $\gamma$ sind unabhängig von $F$ und $R$ :

Diesen Bedingungen genügen zum Beispiel die Polynome, von Faber und die von Fage angegebenen Basisfunktionen. Der dem System $P$ entsprechende Operator von Leontjev wird clurch die Beziehung

$$
D F(z)=\sum_{k=0}^{\infty} d_{k+1} P_{k}(z)
$$

gegeben. Wählen wir $P_{k}(z)=a_{k} z^{k}$, so erhalten wir als Spezialfall den Operator (2).

Es sei $L \in\left[\varrho_{2}, 0\right], L(\lambda)=\sum c_{k} \lambda^{k}$, eine ganze Funktion der Ordnung $\varrho_{2}=\varrho \varrho_{1} /$ $\left(\varrho_{1}-\varrho\right)$ mit dem Typ 0 . Wenden wir uns der Gleichung

$$
\mathcal{M}_{L}\left(F^{\prime}\right):=\sum_{k=0}^{\infty} c_{k} D^{k} F=0
$$

mit $D^{k}=D\left(D^{k-1}\right)$ und $D^{0}=I$ (identischer Operator) zu, dann kann man leicht zeigen, da $B$ die Reihe hierin für jede Funktion $F \in\left[\varrho_{1}, \infty\right)$ konvergiert. Setzen wir zusätzlich voraus, da $L=L_{1} L_{2}$ mit $L_{1}, L_{2} \in\left[\varrho_{2}, 0\right]$ ist, so gilt folgender Satz.

Satz 1: Jede Lösung $F \in\left[\varrho_{1}, \infty\right)$ der Gleichung $\mathscr{M}_{L}(F)=0$ kann man dann und nur dann in der Form

$$
F=\dot{F}_{1}+F_{2} \quad \text { mit } \quad \mathscr{M}_{L_{j}}\left(F_{j}\right)=0 \quad(j=1 ; 2)
$$

darstellen, wenn Funktionen $\varphi_{1}, \varphi_{2} \in\left[\varrho_{2}, 0\right]$ mit jolgender Eigenschaft existieren:

$$
1=L_{1} \varphi_{1}+L_{2} \varphi_{2} \text {. }
$$

2. Einige Hilfsbetrachtungen. Als Hilfsmittel für den Beweis von Satz 1 benötigen wir die folgende in [9] bewiesene Aussage für Differentialgleichungen unendlicher Ordnung in Ableitungen von Gelfond-Leontjev (2).

Satz 2: Es sei die Gleichung

$$
\tilde{\mathscr{M}}_{L}(\dot{F}):=\sum_{k=0}^{\infty} c_{k} D_{j}^{k} F=0
$$

mit der charakteristischen Funktion $L \in\left[\varrho_{2}, 0\right], L(\lambda)=\sum c_{k} \hat{\lambda}^{k}$, gegeben. Jede ihrer Lösungen $\widetilde{F} \in\left[\varrho_{1}, \infty\right)$ ist genau dann in der.Form

$$
\tilde{F}=\tilde{F}_{1}+\tilde{F}_{2} \quad \text { mit } \quad \tilde{\mathscr{M}}_{L}\left(\tilde{F}_{j}\right)=0 \quad(j=1,2)
$$

darstellbar, wenn Funktionen $\varphi_{1}, \varphi_{2} \in\left[\varrho_{2}, 0\right]$ mit der Eigenschaft. (8) existieren.

Im folgenden untersuchen wir die Eigenschaften der Operatoren $D$ und $\mathscr{M}_{L}$. 
Definiert man den Operator $\mathcal{A}$ durch

$$
\mathcal{A} F(z)=\sum_{n=0}^{\infty} d_{n} a_{n} z^{n} \quad \text { für } F \in\left[\varrho_{1}, \infty\right) \text { mit } F(z)=\sum_{n=0}^{\infty} d_{n} P_{n}(z)
$$

dann gilt für den inversen Operator $\mathscr{A}^{-1}$ die Beziehung

$$
\mathcal{A}^{-1} F(z)=\sum_{n=0}^{\infty} \frac{d_{n}}{a_{n}} P_{n}(z) \quad \text { für } F \in\left[\varrho_{1}, \infty\right) \text { mit } F(z)=\sum_{n=0}^{\infty} d_{n} z^{n}
$$

Unter Berückisichtigung von (3) und (4) beweist mán die Stetigkeit der Operatoren $\mathcal{A}$ und $\mathcal{A}^{-1}$ in $\left[\varrho_{1}, \infty\right)$, siehe z. B. [7]. Aus der leicht ersichtlichen Identität $\mathscr{A} D=D_{\text {j }} \mathcal{A}$ erhalten wir für jede Funktion $F \in\left[\varrho_{1}, \infty\right)$ die Beziehung

$$
\mathcal{A} \mathcal{M}_{L}(F)=\tilde{\mathscr{I}}_{L}(\mathcal{A} F)
$$

Wir beweisen nun noch den für den folgenden Abschnitt wichtigen

Hilfssatz: Es seien $f_{1}, f_{2} \in\left[\dot{\varrho}_{2}, 0\right]$ und $f:=f_{1} / f_{2}$ eine ganze Funktion. Dann ist auch $f \in\left[\varrho_{2}, 0\right]$.

Beweis: Wir stützen uns auf die Methode der Monographie [5]. Nach deren Satz 4.3 existiert cine Folge von Kreisen $\Gamma_{k} \doteq\left\{z \in \mathbb{C}:|z|=r_{k}\right\}$ mit $r_{k} \rightarrow \infty, r_{k}<q r_{k-1}$ für ein $q>1$ und mit der Eigenschaft, daß es für.jedes $\varepsilon>0$ eine Zahl $k_{0}(\varepsilon)$ mit $\left|f_{2}(z)\right|>\exp \left(-\varepsilon|z|^{e_{2}}\right)$ für $z \in \Gamma_{k}$ und $k>k_{0}(\varepsilon)$ gibt. Daraus folgt $|f(z)|<\exp \left(\varepsilon|z|^{e_{2}}\right)$ für $z \in \Gamma_{k}, k>k_{1}(\varepsilon) \geqq k_{0}(\varepsilon)$. Wir betrachten jetzt eine beliebige komplexe Zahl $z$ mit $|z|>r_{k_{1}}$. Dann gibt es einen Kreisring $r_{k-1} \leqq|z|<r_{k}, k>k_{1}$; der den Punkt $z$ enthält. Nach dem Maximumprinzip für holomorphe Funktionen gilt $|f(z)| \leqq$ $\max \left\{|f(t)|:|t|=r_{k}\right\}<\exp \left(\varepsilon r_{k}^{e_{1}}\right)$. Wegen $r_{k}<q r_{k-1} \leqq q|z|$ folgt daraus

$$
|f(z)|<\exp \left(\varepsilon q^{e_{3}}|z|^{\ell_{3}}\right) .
$$

Weil dies für beliebiges $\varepsilon>0$ gilt, ergibt sich $f \in\left[\varrho_{2}, 0\right]$

3. Beweis von Satz 1. Es sei (8) erfüllt. Dann erhalten wir unmittelbar aus Satz 2 die additive Zerlegung (10) jeder Lösung $\tilde{F} \in\left[\varrho_{1}, \infty\right)$. Falls $F$ Lösung der Gleichung (6) ist, ergibt sich sofort $\tilde{F}=\mathscr{A} F$ als Lösung der Gleichung (9). Wegen (10) ist $F=\mathcal{A}^{-1} \tilde{F}=\mathcal{A}^{-1} \tilde{F}_{1}+\mathcal{A}^{-1} \tilde{F}_{2}=F_{1}+F_{2}$, wobei $\mathscr{M}_{L_{j}}\left(F_{j}\right)=\mathcal{A}^{-1} \tilde{\mathscr{M}}_{L}\left(\tilde{F}_{j}\right)=0$ $(j=1,2)$ gilt. Also ist die Bedingung (8) hinreichend für (7). Sie ist aber auch notwendig. Dazu setzen wir voraus, da $\beta$ jede Lösung $F \in\left[\varrho_{1}, \infty\right)$ der Gleichung $(6)$ in der Forni (7) darstellbar ist. Für jede beliebige Lösung $\tilde{F}$ der Gleichung (9) ist wegen (11) $F=\mathcal{A}^{-1} \tilde{F}$ Lösung der Gleichung (6). Unter Anwendung von (7) erhalten wir $\tilde{F}=\mathcal{A} F$ $=\mathcal{A} F_{1}+\mathcal{A} F_{2}=\tilde{F}_{1}+\tilde{F}_{2}$, wobei $\mathcal{M}_{L_{s}}\left(F_{j}\right)=0(j=1,2)$ gilt. Nach Satz 2 folgt daraus (8)

Die bekannte Methode von LEontJev (siehe [3]) sowie der obige Hilfssatz und Satz 1 liefern folgende Aussage.

Satz 3: Es sei $L=L_{1} \ldots L_{m}$ mit $L_{j} \in\left[\varrho_{2}, 0\right](1 \leqq j \leqq m)$. Dann läßt sich jede Lösung $F \in\left[\varrho_{1}, \infty\right)$ der Gleichung $(6)$ dann und nur dann in der Form $F=F_{1}+\cdots$ $+F_{m} \cdot$ mit $\mathscr{M}_{L},\left(F_{j}\right)=0(1 \leqq j \leqq m)$ darstellen, wenn Funktionen $\varphi_{1}, \ldots, \varphi_{m} \in\left[o_{2}, 0\right]$ mit der Eigenschaft $1=N_{1} \varphi_{1}+\cdots+N_{m} \varphi_{m}$ und $N_{j}=L / L_{j}(1 \leqq j \leqq m)$ existieren. '

Bemerkung: Wie Satz 3 aus [11] (siehe auch [10]) zeigt, ist die Bedingung (8) äquivalent dazu, daß

$$
\left|L_{1}\left(\lambda_{1}\right)\right|+\left|L_{2}\left(\lambda_{2}\right)\right| \geqq A(\varepsilon) \exp \left(-\varepsilon\left|\lambda_{1}\right|_{2}\right)
$$

für beliebiges $\varepsilon>0$-und eine gewisse Konstante $A(\varepsilon)$ ist. 
4. Beispiele. Das Dargelegte soll durch einige Beispiele illustriert werden.

a) Wir setzen voraus, da $B L_{1}$ und $L_{2}$ Polynome ohne gemeinsame Nullstellen sind. Dann ist der Operator $\mathscr{M}_{L_{1} L_{3}}$ von endlicher Ordnung. Weiterhin wissen wir, daß jede Lösung $F$. der Gleichung (6) in der Form

$$
F(z)=\sum_{k=1}^{N} \sum_{j=0}^{m_{k}-1} c_{k}^{(j)} \frac{\partial^{j}}{\partial \lambda^{j}}\left(\sum_{n=0}^{\infty} \lambda^{n} P_{n}(z)\right)_{\lambda=\lambda_{k}}
$$

darstellbar ist, wobei $m_{k}(k=1, \ldots, N)$ die Vielfachheit der Nullstelle $\lambda_{k}$ des Polynoms $L_{1} L_{2}$ ist. Daraus folgt, da B (7) gilt.

- b) . Es sei $\left\{\lambda_{k}\right\}_{k-1}^{\infty}$-eine monoton wachsende Folge von nichtnegativen Zahlen, die der Eigen-schaft

$$
n / \lambda_{n} e_{2} \rightarrow 0 \quad \text { für } \quad n \rightarrow \infty \quad\left(\varrho_{2}>1\right)
$$

genügt. Außerdem sei $m>\varrho_{2}$ eine natürliche Zahl. Wir setzen voraus, daß sich die Kreise $\left\{z:\left|z-\lambda_{n} E^{k}\right| \leqq d \lambda_{n}{ }^{1-\ell},\right\}(k=0, \ldots, m-1 ; n \in \mathbb{N})$ mit $E^{\prime}=\exp (2 \pi \mathrm{i} / m)$ und einem gewissen $d>0$ nicht schneiden. Wir betrachten jetzt die ganze Funktion $L=L_{1} L_{2}$ mit

$$
L_{1}\left(\lambda_{1}\right)=\prod_{n=1}^{\infty}\left(1-\lambda^{m} /\left(\lambda_{n}^{\prime}\right)^{m}\right), \quad L_{2}\left(\lambda_{1}\right)=\prod_{n=1}^{\infty}\left(1-\lambda^{m} /\left(\lambda_{n}^{\prime \prime}\right)^{m}\right)
$$

und $\left\{\lambda_{n}\right\}=\left\{\lambda_{n}{ }^{\prime}\right\} \cup\left\{\lambda_{n}{ }^{\prime \prime}\right\}$. Aus (14) folgt unmittelbar $L_{1}, L_{2}, L \in\left[e_{2}, 0\right]$. Nach Satz 1.2.7 aus [4] ist $\left|L_{2}\left(\lambda_{n}{ }^{\prime} E^{k}\right)\right|>\exp \left(-\delta^{\prime}\left|\lambda_{n}{ }^{\prime}\right|{ }^{\prime}\right)\left(k=0, \ldots, m-1 ; n>n_{0}(\delta)\right)$. Satz 7 aus [1: S. 36] (siehe auch $\left[4:\right.$ S. 49]) sichert die Existenz einer Funktion $\varphi \in\left[\varrho_{2}, 0\right]$ mit der Eigenschaft $\varphi\left(\lambda_{n}{ }^{\prime} E^{k}\right)=$ $1 / L_{2\left(\lambda_{n}^{\prime} E^{k}\right)}(k=0, \ldots, m-1 ; n \in \mathbb{N})$. Damit erhalten wir das Verschwinden der Funktion $\varphi L_{2}-1$ in allen Nullstellen von $L_{1}$ und daraus sofort, daß $\psi=\left(\varphi L_{2}-1\right) / L_{1}$ eine ganze Funktion ist. Nach dem Hilfśsatz ist $\psi \in\left[\varrho_{2}, 0\right]$, und damit ist (8) erfüllt.

c) Im letzten Beispiel wollen wir zeigen, da $B$ auch Funktionen $L_{1}, L_{2} \in\left[\varrho_{2}, 0\right]$ existieren, für welche keine Funktionen $\varphi_{1}, \varphi_{2} \in\left[\varrho_{2}, 0\right]$ mit (8) gefunden werden können. Dieser Art sind z. B. die Funktionen (15) mit $\lambda_{k}^{\prime}=k^{2 / e^{2}}, \lambda_{k}^{\prime \prime}=k^{2 / e}+\delta_{k}$ und $\delta_{k}=\exp \left(-k^{3}\right)$. Angenommen die Vermutung ist falsch. Dann folgt aus (13) mit einer positiven Konstanten $A(\varepsilon)$

$$
\left|L_{2}\left(\lambda_{k}{ }^{\prime}\right)\right|>A(\varepsilon) \exp \left[-\varepsilon\left|\lambda_{k}{ }^{\prime}\right| e^{*}\right]
$$

Wegen $L_{2}^{\prime} \in\left[\varrho_{2}, 0\right]$ erhalten wir

$$
\left|L_{2}\left(\lambda_{k}^{\prime}\right)\right|=\left|\int_{\lambda_{k}^{\prime}}^{\lambda_{k}^{\prime \prime}} L_{2}^{\prime}(t) d t\right| \leqq\left|\lambda_{k}^{\prime \prime}-\lambda_{k}^{\prime}\right| A_{1}(\varepsilon) \exp \left(\varepsilon\left|\lambda_{k}{ }^{\prime \prime}\right| \mathbf{e}^{2}\right),
$$

und mit (16) folgt daraus $\left|\lambda_{k}{ }^{\prime \prime}-\lambda_{k}^{\prime}\right| \exp \left(2 \varepsilon\left|\lambda_{k}{ }^{\prime \prime}\right| \rho^{s}\right)>A_{2}(\varepsilon)>0$. Dieser Widerspruch beweist die Gültigkeit der Vermutung.

\section{LITEIRATUR}

[1] ЛЕонтьев, А. Ф.: Рядњ полиномов Дирихле и их обобщения. Москва: Изд-во Наука 1951 .

[2] ЛЕонтьев, А. Ф.: Об одном функциональном уравнении. Изв. Акад: Наук СССР, Сер. мат., 29 (1965), 725-756.

[3] JI єонтьев, А. Ф.: Об одном применении интерполяционного метода. Мат. заметки $1 \mathrm{~S}(1975), 735-752$.

[4] ЈI ғонтьеВ, А. Ф.: Ряды экспонент. Москва: Изд-во Наука 1976.

[5] Л контьев, А. Ф.: Целые функции. Ряды әкспонент. Москва: Изд-во Наука 1983.

[6] Matzudra, S.: Factorization of differential operators and decomposition of homogeneous equations. Osaka J. Math. 15 (1963), $213 \div 231$. 
[7] Мустафин, Р. Ф., и В. П. ПодПорин: Спектральный синтез в пространстве целых функций ограниченного роста. Иав. высш. уч. зав., Математика 290 (1986), $84-87$.

[8] Напалков, В. В.: Факторизация оператора типа свертки. Мат. Заметки 15 (1974), $165-171$.

[9] Тимофевв, А. Ю:: Представление решения функционального уравнения в виде суммы двух решенй. В кн.: Исследования по теории аппроксимации функций (отв.ред.: В. В. Напалков). Уфа: Башк. филиал Акад. наук СССР 1981, стр. 92-100.

[10] ТимофеЕв, А.. Ю.: О представлении решения уравнения бесконечного порядка $\mathrm{B}$ виде суммы двух решенин. Мат. заметки 31 (1982), 245-256.

[11].ТмофеЕв, А. Ю.: О представлении решения операторного уравнения в виде суммы решенић. В кн.: Исследования по теории операторов (отв. ре́д.: В. В.'Напалков). Уфа: Башк. филиал Акад. наук СССР 1988, стр : 146-152.

Manuskripteingang: 18.01. 1988

\section{VERFASSER:}

\section{Dr. Alexej Timofeev}

Lehrstuhl für Höhere Mathematik der Staatlichen Universität .

Oktjabrskij prospekt 55

UdSSR - 167001 Syktywkar

\section{Buchbesprechung.}

A. Pietsch : Eigenvalues and s-Numbers (Math. u. Anw. in Phys. u. Techn.: Bd. 43). Leipzig: Akad. Verlagsges. Geest \& Portig 1987, pp. 360.

Nach "Operator Ideals" liegt mit diesem Buch eine neue Monographie von A. Pietsch vor, die auch inhaltlich als eine Fortsetzung angesehen werden kann. Von den im ersten Buch angedeuteten Anwendungen der Theorie der Operatorenideale erweisen sich deren Begriffe und Methoden besonders im Zusammenhang mit Untersuchungen über Eigenwertverteilungen von Integraloperatoren als überaus fruchtbar. Durch die Arbeiten einer Reihe von Autoren sind in den letzten zehn Juhren viele neue und wertvolle Ergebnisse gefunden worden, so dnß sich inzwischen eine gehaltvolle eigenständige Theorie entwickelt hat, zu der es bisher nur ein Buch (H. König: Eigenvalue Distribution of Compact Operators. Basel: Birkhäuser Verlag 1986) gab. Anliegen des Autors ist es, eine geschlossene Darstellung dieser Resultate zu bringen und aufbauend auf eigenen Ergebnissen - eine einheitliche Theorie zu präsentieren. Dies gelingt auf eine überzeugende Weise. Konsequent wird der gesamte Stoff streng deduktiv aufgebaut und formalisiert. Das führt zu einer starken Algebraisierung der Darstellung, durch die die durchgehenden und Beweisideen klar herausgearbeitet werden können. Diese Konsequenz ist auch deutlich im Stil des gesamten Buches zu spüren, der durch seine nahezu perfekte * Okonomie auf den ersten Blick vielleicht recht trocken wirkt. So lesen sich manche Teile wie ein Kursbuch, wenn umfangreiche Beweise in selbständige, mehrfach nutzbare Lemmata mit eigenständigen Aussagen zerlegt werden, die anschließend der Reihe nach aufgerufen werden. 